\title{
An Efficient Scheme for Nonlinear Modeling and Predistortion in Mixed-Signal Systems
}

\author{
Heinz Koeppl and Peter Singerl
}

\begin{abstract}
A novel identification and predistortion scheme of weakly nonlinear systems for mixed-signal devices, which takes into account practical implementation aspects, is presented. It is well known that for the identification of weakly nonlinear systems, despite the spectral regrowth, it suffices to sample the input-output (I/O) data of the system at the Nyquist rate of the input signal. Many applications such as linearization and mixed-signal simulations require system models at a higher sampling rate than Nyquist. Up to now, the construction of such high-rate models has been done by oversampling the corresponding I/O data. This leads to high computational complexity, ill posedness of the estimation, and high demand on the analog-to-digital-converter sampling rate for the implementation. This brief discusses an efficient way to obtain high-rate models and predistorters from low-rate models and shows the validity of the proposed scheme for a very-high-speed-digital-subscriber-line power amplifier, where an adjacent channel power supression of $20 \mathrm{~dB}$ is achieved.
\end{abstract}

Index Terms-Adaptive equalizers, amplifier distortion, data communication, nonlinear distortion, nonlinear filters, predistortion, Volterra series.

\section{INTRODUCTION}

$\mathbf{T}$ O BUILD mixed-signal devices with low-power consumption, an increasing trend in system design is to utilize cheap and power-efficient architectures in the analog part of the device and transfer as much of the processing as possible to the digital part of the device. Operating power amplifiers (PAs) in their saturated region or changing from class- $\mathrm{A} / \mathrm{B}$ to class- $\mathrm{C}$ or class-D architectures can yield a large gain in efficiency. The price paid for higher efficiency in general is that the stronger nonlinearity of the PA causes in-band distortion, which degrades the bit-error performance. It also causes out-of-band spectral regrowth, which leads to adjacent channel interference. Newer modulation formats such as wideband code-division multiple access (WCDMA), orthogonal frequency-division multiplexing (OFDM), and discrete multitone modulation (DMT) are especially vulnerable to nonlinearities due to their high peak-to-average power ratio (PAR). In order to comply with the spectral masks imposed by regulatory bodies and to reduce the bit-error rate, linearization of the analog part is frequently deployed. The most powerful and efficient linearization technique is digital predistortion [1]-[4]. Fig. 1 depicts a simplified block diagram of a communications transmitter. The

Manuscript received December 9, 2005; revised February 24, 2006. This paper was recommended by Associate Editor T. Saito.

H. Koeppl is with the Department of Electrical Engineering and Computer Sciences, University of California Berkeley, Berkeley, CA 94720 USA (e-mail: heinz.koeppl@berkeley.edu).

P. Singerl is with the Christian Doppler Laboratory for Nonlinear Signal Processing, Graz University of Technology, 8010 Graz, Austria. He is also with Infineon Technologies, 9500 Villach, Austria.

Digital Object Identifier 10.1109/TCSII.2006.882232

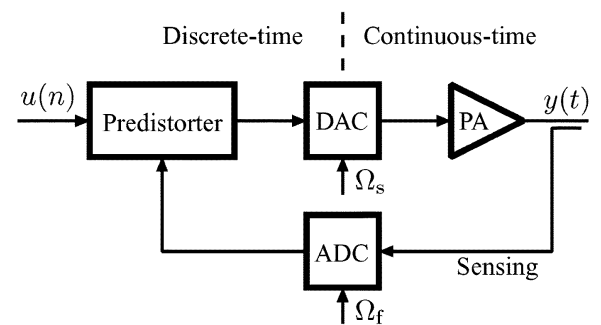

Fig. 1. Block diagram of a communications transmitter with digital predistortion for the linearization of the PA.

digital predistorter acts on the input signal by using a nonlinear operator, which is ideally the inverse nonlinearity of the PA, to obtain an overall linear response.

To compensate the out-of-band spectral components caused by the nonlinearity, the predistorter has to be operated at a sufficiently high sampling frequency $\Omega_{\mathrm{s}}$, which is usually $2 P$ times higher than the transmission signal bandwidth $B$, where factor $P$ denotes the highest order of significant nonlinearity in the PA. Because the nonlinear behavior of the PA is in general unknown, one applies a feedback path in Fig. 1 in order to either identify the amplifier and compute the inverse (predistorter) or identify immediately the predistorter [1], [5]. It is well known that the identification of a Volterra system can be accomplished at a sampling rate that is just twice the input signal bandwidth [6]. Based on this result, we upsample the low-rate identified Volterra kernels. Thereby, we can reduce the sampling rate of the feedback path from $\Omega_{\mathrm{s}}$ to $\Omega_{\mathrm{f}}=\Omega_{\mathrm{s}} / P$, compared to the traditional approach.

While [7] considers the kernel upsampling and predistortion of baseband Volterra systems for mobile radio applications only, this brief lays out the general methodology for the class of weakly nonlinear systems. The subsequent results are obtained for nonlinear models that can be described by an additive decomposition of a dominant linear part and a perturbative nonlinear part. Where necessary, we will assume a representation of the nonlinear model in terms of its Volterra series, which covers the entire class of weakly nonlinear systems. Belonging to this class are all approximate models for weakly nonlinear systems such as the Wiener model, the Hammerstein model, and the "linear-nonlinear-linear" (LNL) structure [8]. A thorough introduction to Volterra series and their classical input-output (I/O) relation is provided in [9].

For the sake of clarity, the following notation and definitions are used throughout the rest of this brief. A continuous-time weakly nonlinear system, often representing nonlinear PAs, will be referred to as the "system" $\mathrm{V}: u \mapsto y$, with $u, y \in$ $\mathrm{L}_{2}\left(\mathbb{R}_{+}\right)$, where $\mathrm{L}_{2}\left(\mathbb{R}_{+}\right)$denotes the space of square integrable signals and $\mathbb{R}_{+}$is the set of positive reals. Its associated set of 


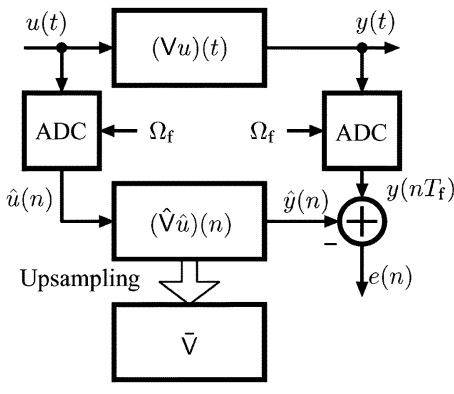

Fig. 2. System identification setup at $\Omega_{\mathrm{f}}$ and the generation of a high-rate model $\bar{V}$ for predistortion and modeling purposes.

Laplace-domain Volterra kernels is $\left\{V\left(s_{1}, \ldots, s_{p}\right)\right\}$ with $p=$ $1, \ldots, P$, where the order of homogeneity of a kernel is not given explicitly but can be read off from the number of its arguments. A discrete-time Volterra model operating at the Nyquist rate $\Omega_{\mathrm{f}}$ of the input signal will be referred to as the "low-rate model" $\hat{V}: \hat{u} \mapsto \hat{y}$ with $\hat{u}, \hat{y} \in \ell_{2}\left(\mathbb{Z}_{+}\right)$, where $\ell_{2}\left(\mathbb{Z}_{+}\right)$denotes the space of square summable infinite sequences and $\mathbb{Z}_{+}$is the set of positive integers. Its upsampled version operating at an integer multiple of $\Omega_{\mathrm{f}}$, especially at the Nyquist rate of the output signal $\Omega_{\mathrm{s}}$, will be termed the "upsampled model" $\overline{\mathrm{V}}: \bar{u} \mapsto \bar{y}$, with $\bar{u}, \bar{y} \in \ell_{2}\left(\mathbb{Z}_{+}\right)$. Their associated $\mathcal{Z}$ transform kernels are $\left\{V\left(z_{1}, \ldots, z_{p}\right)\right\}$ and $\left\{V\left(z_{1}, \ldots, z_{p}\right)\right\}$ with $p=1, \ldots, P$, respectively. With a slight abuse of notation but for the sake of conciseness and without loss of generality in case of bandlimited input signals [6], we also associate with the system $\mathrm{V}$ its exact equivalent discrete-time system $\ell_{2}\left(\mathbb{Z}_{+}\right) \mapsto \ell_{2}\left(\mathbb{Z}_{+}\right)$at $\Omega_{\mathrm{s}}$ with kernels $\left\{V\left(z_{1}, \ldots, z_{p}\right)\right\}$. Throughout this brief, we restrict ourselves to a frequency-domain analysis, i.e., $s_{k}=j \Omega_{k}$ and $z_{k}=e^{j \theta_{k}}$ with $k=1, \ldots, p$.

This brief is organized as follows. In Section II, we introduce the idea of Volterra kernel upsampling and its application to nonlinear system modeling. Section III presents the predistortion architecture and briefly discusses its relation to the $p$ th-order inverse method [10]. The application of the kernel upsampling method to the considered predistortion architecture and its implication in terms of efficiency is discussed in Section IV. The performance of the presented scheme is evaluated in Section V using a model of a PA used in a very high speed digital subscriber line (VDSL) communications systems [11], while Section VI draws conclusions.

\section{VOLTERRA KERNEL IDENTIFICATION AND UPSAMPLING}

The question posed in this section is the following: Can we identify a low-rate model of the system and then "upsample" the model such that it is valid for higher sampling rates? The identification setup is depicted in Fig. 2. According to [6], one can find a low-rate model $\hat{V}$ that reproduces exactly the sampled output $y\left(n T_{\mathrm{f}}\right)$ of the system $\vee$ with $T_{\mathrm{f}}=2 \pi / \Omega_{\mathrm{f}}$.

The applied upsampling method translates from careful consideration of the upsampling procedure for linear systems, whereas throughout the text, upsampling is defined as zero-padding without a following interpolation. The multidimensional zero-padding of Volterra kernels corresponds to a frequency scaling and a periodic extension of $\left\{\hat{V}\left(z_{1}, \ldots, z_{p}\right)\right\}$. Thus, the kernels of the upsampled model are obtained as

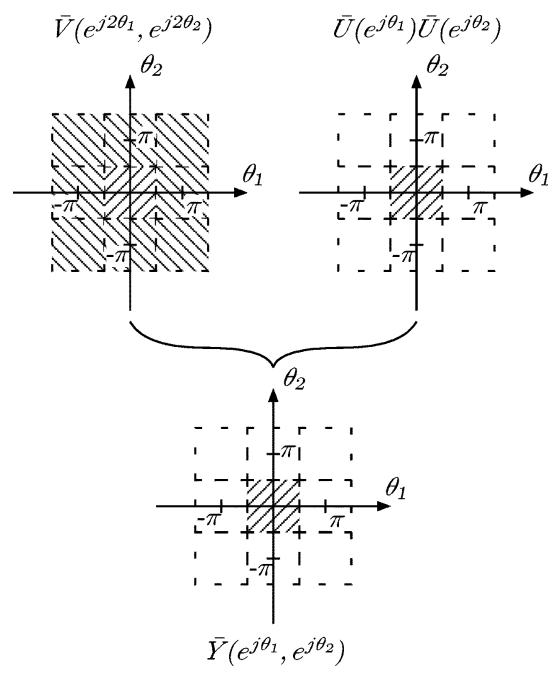

Fig. 3. Two-dimensional zero-padding and spectral masking by the high-rate input signal $\bar{U}\left(e^{j \theta_{1}}\right) \bar{U}\left(e^{j \theta_{2}}\right)$ for a second-order Volterra kernel. The shaded areas indicate the nonzero frequency-domain kernel.

$\hat{V}\left(z_{1}, \ldots, z_{p}\right)=\bar{V}\left(z_{1}^{P}, \ldots, z_{p}^{P}\right)$. Note that the periodic extension implies that the kernels of the obtained upsampled model coincide with those of the system $\mathrm{V}$ only over a limited frequency region, i.e., $\bar{V}\left(e^{j \theta_{1}}, \ldots, e^{j \theta_{p}}\right)=V\left(e^{j \theta_{1}}, \ldots, e^{j \theta_{p}}\right)$ for $\theta_{k} \in[-\pi / P, \pi / P)$, with $\theta_{k}=2 \pi \Omega_{k} / \Omega_{\mathrm{S}}$ and $k=1, \ldots, p$. However, at the high rate, the interpolated input signal $\bar{U}\left(e^{j \theta}\right)$ is nonzero only for $\theta \in[-\pi / P, \pi / P)$, such that the system responses of $\bar{V}$ and $V$ are identical. This process is illustrated for the response of a homogeneous second-order Volterra system in Fig. 3. Thus, the aforementioned question can be answered positively. Note that to upsample a Volterra model, one solely has to replace each delay element $z^{-1}$ by $z^{-P}$.

\section{Predistortion Architecture}

The predistorter $\mathrm{P}$ precedes the PA in order to distort the incoming signal to obtain ideally an overall linear system response of the cascade of predistorter and PA. In [4], it is shown that for a certain class of nonlinear systems, an exact inverse exists. However, for most systems, we are not able to find an exact inverse [10]. Researchers have found different approximate system inverses that are based either on PA modeling and predistorter calculation or on direct predistorter estimation [1]-[3], [10].

In this brief, we focus on a simple predistortion architecture $P$ proposed in [1], which is composed of a parallel structure of delay operator $z^{-\delta}$ and a cascade of the purely nonlinear part $\breve{V}_{n}$ with the inverse of the linear part $\breve{V}_{1}$ of an operator $\breve{V}$, i.e., $\mathrm{P} \equiv z^{-\delta}+\breve{V}_{1}^{-1} \circ \breve{V}_{\mathrm{n}}$, where "o" denotes the composition of operators. The set of Volterra kernels of $\breve{V}$, which is denoted as $\left\{V\left(z_{1}, \ldots, z_{p}\right)\right\}$, with $p=1, \ldots, P$ is determined in the following: The notation that the operators precede their arguments is used throughout this brief. The architecture of $\mathrm{P}$ as part of the overall system can be shown in Fig. 4. The composition of the predistorter and the system

$$
\mathrm{L} \equiv\left(\mathrm{V}_{\mathrm{I}}+\mathrm{V}_{\mathrm{n}}\right) \circ\left(z^{-\delta}+\breve{\mathrm{V}}_{\mathrm{I}}^{-1} \circ \breve{V}_{\mathrm{n}}\right)
$$

yields a linearization if $\left\|\mathrm{V}_{\mathrm{n}}\right\| \ll\left\|\mathrm{V}_{\mathrm{l}}\right\|$ with $\breve{\mathrm{V}}_{\mathbf{l}}=-z^{\delta} \circ \mathrm{V}_{\mathrm{I}}$ and $\breve{V}_{n}=V_{n}$, where the operator norm corresponds to the signal 


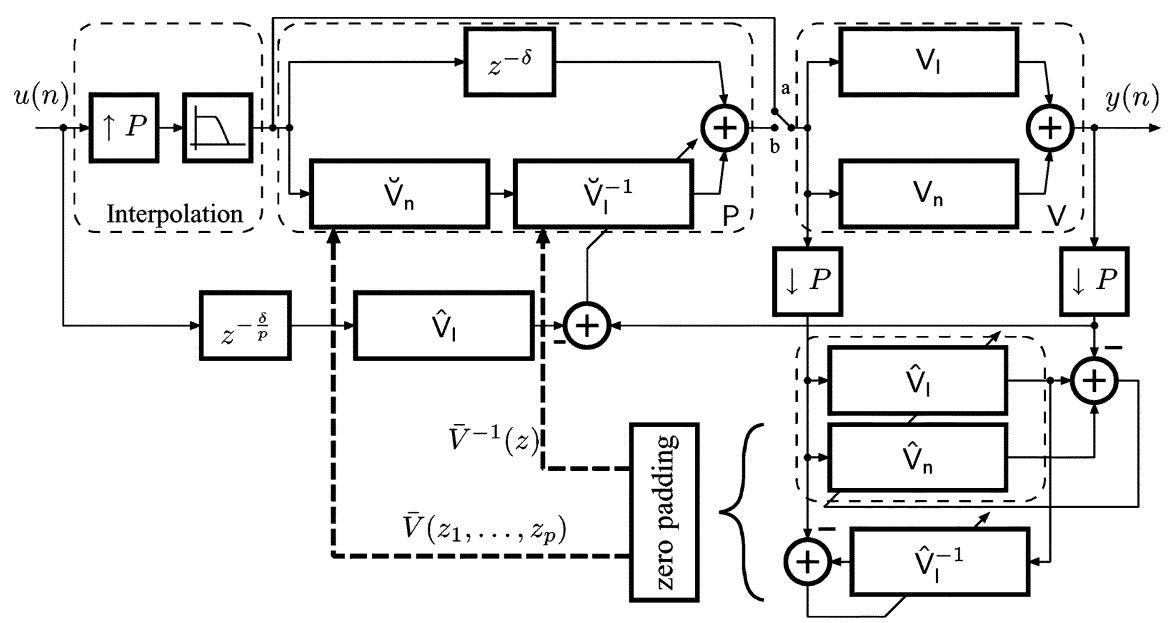

Fig. 4. Multirate digital predistortion architecture $\mathrm{V} \circ \mathrm{P}$ composed of a high-rate system $\mathrm{V}$, a high-rate predistorter $\mathrm{P}=z^{-\delta}+\breve{V}_{\boldsymbol{I}}^{-1} \circ \breve{V}_{\mathrm{n}}$, with an optional low-rate adaptation of the linear operator $\breve{V}_{\mathbf{l}}$, a low-rate system identification $\hat{\mathrm{V}}$, and Volterra kernel upsampling (zero padding); switch position a for identification and position $\mathrm{b}$ for predistortion; and $\delta=n P, n \in \mathbb{Z}_{+}$.

norm with $\left\|\mathrm{V}_{\mathbf{l}}\right\| \equiv \sup _{\|u\|<1}\left\|\mathrm{~V}_{\mathbf{l}} u\right\|$. Qualitatively, this becomes clear by decomposing (1) as

$$
\mathrm{L}=z^{-\delta} \circ \mathrm{V}_{\mathbf{I}}+\mathrm{V}_{\mathbf{I}} \circ \breve{V}_{\mathbf{I}}^{-1} \circ \breve{V}_{\mathrm{n}}+\mathrm{V}_{\mathrm{n}} \circ\left(z^{-\delta}+\breve{\mathrm{V}}_{\mathbf{I}}^{-1} \circ \breve{V}_{\mathrm{n}}\right)
$$

where we used the fact that the delay operator commutes with all time-invariant operators. The preceding assumption on weak nonlinearity implies $\left\|\mathrm{V}_{\mathrm{n}} \circ \breve{V}_{\mathrm{I}}^{-1} \circ \breve{V}_{\mathrm{n}}\right\| \ll\left\|\mathrm{V}_{\mathrm{n}}\right\|$, such that we can approximate the last term in (2) as $\mathrm{V}_{\mathrm{n}} \circ\left(z^{-\delta}+\breve{\mathrm{V}}_{\mathbf{l}}^{-1} \circ \breve{V}_{\mathrm{n}}\right) \approx$ $\mathrm{V}_{\mathrm{n}} \circ z^{-\delta}$. Thus, the overall system response is rendered as $\mathrm{L} \approx$ $z^{-\delta} \circ \mathrm{V}_{\mathbf{l}}$.

A quantitative analysis of the linearization performance of the architecture can be done if one employs the Volterra series representation of the involved operators. Subsequently, the set of kernels $\left\{L\left(z_{1}, \ldots, z_{p}\right)\right\}$ for $p=0,1,2, \ldots, P^{2}$ of $L$ is expressed in terms of the kernels of the operators according to the composition in (1). For clarity of presentation, we indicate the order of homogeneity of a Volterra kernel by an additional subscript in the following derivation: Allowing for nonminimum-phase systems with $\breve{V}_{1}(z) \breve{V}_{1}^{-1}(z)=z^{-\delta}$, the application of the $\mathcal{Z}$-domain cascade rule [9] for discrete-time Volterra series to (1) yields

$$
\begin{gathered}
L_{p}\left(z_{1}, \ldots, z_{p}\right) \\
=\sum_{n=1}^{p} \underbrace{\sum_{m_{1}=1} \cdots \sum_{m_{n}=1}}_{\omega(n)=p}\left[V_{n}\left(z_{1} \cdots z_{\omega(1)}, \ldots, z_{\alpha(n)} \cdots z_{\omega(n)}\right)\right. \\
\times \prod_{k=1}^{n} \breve{V}_{1}^{-1}\left(z_{\alpha(k)} \cdots z_{\omega(k)}\right) \\
\left.\times \breve{V}_{m_{k}}\left(z_{\alpha(k)}, \ldots, z_{\omega(k)}\right)\right]
\end{gathered}
$$

with the lower and upper index bound functions $\alpha(k) \equiv$ $\alpha\left(m_{1}, \ldots, m_{k-1}\right)=1+\sum_{j=1}^{k-1} m_{j}$ and $\omega(k) \equiv$ $\omega\left(m_{1}, \ldots, m_{k}\right)=\sum_{j=1}^{k} m_{j}$, respectively, with $\alpha(1)=1$. Separating the terms in (3) for $n=1$ and $n=p$ gives

$$
\begin{aligned}
& L_{p}\left(z_{1}, \ldots, z_{p}\right) \\
& =V_{1}\left(z_{1} \cdots z_{p}\right) \breve{V}_{1}^{-1}\left(z_{1} \cdots z_{p}\right)
\end{aligned}
$$

$$
\begin{gathered}
\times \breve{V}_{p}\left(z_{1}, \ldots, z_{p}\right)+V_{p}\left(z_{1}, \ldots, z_{p}\right)\left(z_{1} \cdots z_{p}\right)^{-\delta} \\
+\sum_{\omega(n)=p}^{\sum_{n=2}^{p-1} \ldots \sum_{m_{n}=1}}\left[V_{n}\left(z_{1} \cdots z_{\omega(1)}, \ldots, z_{\alpha(n)} \cdots z_{\omega(n)}\right)\right. \\
\times \prod_{k=1}^{n} \breve{V}_{1}^{-1}\left(z_{\alpha(k)} \cdots z_{\omega(k)}\right) \\
\left.\times \breve{V}_{m_{k}}\left(z_{\alpha(k)}, \ldots, z_{\omega(k)}\right)\right]
\end{gathered}
$$

where the first two summands in (4) equalize if the linear frequency-domain kernel is

$$
\breve{V}_{1}(z)=-V_{1}(z) z^{\delta}
$$

and the kernels for $p \geq 2$ are

$$
\breve{V}_{p}\left(z_{1}, \ldots, z_{p}\right)=V_{p}\left(z_{1}, \ldots, z_{p}\right) .
$$

Thus, the system with an adjusted predistorter results in the following Volterra kernels:

$$
\begin{aligned}
& L_{1}(z)=V_{1}(z) z^{-\delta} \\
& L_{2}\left(z_{1}, z_{2}\right)=0 \\
& L_{3}\left(z_{1}, z_{2}, z_{3}\right) \\
& =-\left[V_{2}\left(z_{1} z_{2}, z_{3}\right) V_{1}^{-1}\left(z_{1} z_{2}\right) V_{2}\left(z_{1}, z_{2}\right)\right. \\
& \left.+V_{2}\left(z_{1}, z_{2} z_{3}\right) V_{1}^{-1}\left(z_{2} z_{3}\right) V_{2}\left(z_{2}, z_{3}\right)\right]\left(z_{1} z_{2} z_{3}\right)^{-\delta} \\
& L_{p}\left(z_{1}, \ldots, z_{p}\right) \\
& =-\sum_{n=2}^{p-1} \underbrace{\sum_{m_{1}=1} \cdots \sum_{m_{n}=1}}_{\omega(n)=p}\left[V_{n}\left(z_{1} \cdots z_{\omega(1)}, \ldots, z_{\alpha(n)} \cdots z_{\omega(n)}\right)\right. \\
& \times \prod_{k=1}^{n}\left(z_{\alpha(k)} \cdots z_{\omega(k)}\right)^{-\delta} \\
& \times V_{1}^{-1}\left(z_{\alpha(k)} \cdots z_{\omega(k)}\right) \\
& \left.\times V_{m_{k}}\left(z_{\alpha(k)}, \ldots, z_{\omega(k)}\right)\right] \text {. }
\end{aligned}
$$


Although perfect equalization is only possible for the secondorder Volterra kernel $L_{2}\left(z_{1}, z_{2}\right)$, the resulting distortion of the predistorted PA output signal is much lower compared to the noncompensated PA if $\left\|\mathrm{V}_{\mathrm{n}}\right\| \ll\left\|\mathrm{V}_{\mathrm{l}}\right\|$ because all the kernels $L_{p}\left(z_{1}, \ldots, z_{p}\right)$ for $p \geq 3$ in (7) incorporate the multiplicative inverse of the linear kernel $V_{1}(z)$.

To establish a relation to the more general linearization method of the $p$ th-order inverse [10], we consider the special case of the second-order inverse for a system described by the operator decomposition $V=V_{1}+V_{2}+V_{h}$, where the operator $V_{\mathrm{h}}$ resembles all operators with a homogeneity order of $p>2$. The predistorter $\tilde{\mathrm{P}}$ based on the second-order inverse, where the linear frequency response is not equalized, gives $\tilde{P}=z^{-\delta} \circ\left(1-V_{1}^{-1} \circ V_{2}\right)$, while according to (1), the applied predistorter is $\mathrm{P}=z^{-\delta} \circ\left[1-\mathrm{V}_{1}^{-1} \circ\left(\mathrm{V}_{2}+\mathrm{V}_{\mathrm{h}}\right)\right]$. From that, it is obvious that for a second-order nonlinear system $V$, the two predistortion schemes coincide. The comparison of the two schemes in case of a third-order inverse of a system $\mathrm{V}=\mathrm{V}_{1}+\mathrm{V}_{2}+\mathrm{V}_{3}+\mathrm{V}_{\mathrm{h}}$ will point out an essential difference, of which the important consequences for a low-rate implementation are discussed in Section IV. The predistortion operator $\tilde{P}$ based on the third-order inverse [10] is given by

$$
\begin{aligned}
\tilde{\mathrm{P}}=z^{-\delta} \circ\left\{1-\mathrm{V}_{1}^{-1} \circ[\right. & \mathrm{V}_{2} \circ \mathrm{V}_{1}^{-1} \circ \mathrm{V}_{2} \\
& \left.\left.+\mathrm{V}_{2} \circ\left(1-\mathrm{V}_{1}^{-1} \circ \mathrm{V}_{2}\right)+\mathrm{V}_{3}\right]\right\} .
\end{aligned}
$$

\section{PREDISTORTION AND UPSAMPLING}

As shown in Section II, the low-rate model $\hat{V}$ can be upsampled to a higher rate such that it exactly reproduces the output of the system $\mathrm{V}$. The natural question posed in this section is whether or not one can apply the upsampled model of Section II in the predistortion architecture of Section III with $\breve{V}(z)=-\bar{V}(z) z^{\delta}$ and $\breve{V}\left(z_{1}, \ldots, z_{p}\right)=\bar{V}\left(z_{1}, \ldots, z_{p}\right)$ such that it yields an identical result as the corresponding application of the kernels of $V$ in (5) and (6). As the model is able to reproduce the exact output of the system, one is tempted to answer this question positively. Unfortunately, they do not perform in the same way when applied to the predistortion architecture. To see this, note first that the kernels of the upsampled model and the system coincide only over the bandwidth of the input signal $\bar{U}\left(e^{j \theta}\right)$. Reconsideration of the structure of the predistorter $\mathrm{P}=z^{-\delta}+\breve{V}_{1}^{-1} \circ \hat{V}_{\mathrm{n}}$ reveals that due to the spectral regrowth of $\breve{V}_{n}$, the linear operator $\breve{V}_{I}^{-1}$ acts on a signal with a bandwidth that is in general $P$ times the input signal bandwidth. Thus, the output signal of the upsampled version of $\breve{V}_{I}^{-1}$ differs from the correct version given in (5) and (6) by an amount that depends on the flatness of the linear frequency response of the system. The more flat the response, the less the effect of the periodic extension in the upsampled version outside the input signal bandwidth. To correct the periodic extensions of $\breve{V}(z)$, one can apply a low-rate adaptation, where the initialization is taken to be the upsampled response $\bar{V}(z)$. The adaptation is depicted in Fig. 4.

In this context, an important property of the applied predistortion architecture $\mathrm{P}=z^{-\delta}+\breve{V}_{1}^{-1} \circ \breve{V}_{\mathrm{n}}$ becomes evident. As the operator $\breve{V}_{\mathrm{n}}$ with the kernels $\left\{\bar{V}\left(z_{1}, \ldots, z_{p}\right)\right\}$ with $p=$ $2, \ldots, P$ operates on the input signal bandwidth, its response is identical to the response of $\breve{V}_{\mathbf{n}}$ with $\left\{V\left(z_{1}, \ldots, z_{p}\right)\right\}$ and thus requires no adaptation. This feature allows for efficient low-rate adaptive predistortion, where only the few parameters of $\breve{V}(z)$ need to be adapted, while the mass of parameters from the higher order frequency responses requires no adaptation. This is contrary to the application of the upsampled kernels $\left\{\bar{V}\left(z_{1}, \ldots, z_{p}\right)\right\}$ to the $p$ th-order inverse architecture.

As an example, consider the summands in (8) for the thirdorder inverse. In the case of upsampled kernels, for every composition of operators where a nonlinear operator $\bigvee_{\mathrm{k}}$ with $k>1$ is preceding one or more operators, an adaptation of all consecutive operators following $V_{k}$ is necessary to yield an equivalent performance to a third-order inverse utilizing the correct high-rate kernels of the system $V$.

The overall system including the identification of the low-rate model $\hat{V}$ of the system $V$, predistorter $P$ with its optional adaptation, and the kernel upsampling is shown in Fig. 4. The switch in Fig. 4 indicates that for the initial identification of low-rate model $\hat{V}$, the predistorter is bypassed. Note that simultaneous identification and predistortion is in general not possible due to the spectral regrowth at the output of the predistorter.

\section{ApPlication AND Simulation Results}

To evaluate the performance of the proposed low-rate predistortion scheme, the linearization of a PA for a wire-line transmission system is performed. The system $V$ is taken to be a high-rate discrete-time model of a PA for a VDSL analog front end [11] that complies with the distortion ratios of the standard [12]. To be able to apply predistortion over a realistic distortion range, the circuit was mistuned to yield a harmonic distortion ratio of $40 \mathrm{~dB}$. The model has been obtained by fitting a simple fifth-order Volterra model to the I/O measurement of a transistor-level circuit simulation. The standard four-band VDSL system can utilize a bandwidth of up to $12 \mathrm{MHz}$, where each band is DMT modulated. The frequency allocation for this simulation according to the standard [12] (Band Plan 998) consists of two downstream bands, each having a bandwidth of 2.59 $\mathrm{MHz}$ and located at center frequencies of 2.225 and $6.885 \mathrm{MHz}$. Following the exposition of the previous sections, the sampling rate of digital predistortion for that system has to be at least $\Omega_{\mathrm{s}}=2 \pi \times 81.8 \mathrm{MHz}$. Conventionally, this directly translates to the sampling requirements of the ADC in the feedback path (refer to Fig. 1) used for the parameterization of the predistorter. The proposed approach requires an ADC sampling rate of only $\Omega_{\mathrm{f}}=2 \pi \times 16.36 \mathrm{MHz}$.

The following results are all obtained by averaging over 50 different DMT signals with identical PAR but randomly chosen phase constellation: That constant PAR corresponds to the average PAR occurring if the phase constellations are drawn from the uniform distribution $\mathcal{U}[0,2 \pi)^{N}$, with $N$ being the number of carriers. In Fig. 5, the average power spectrum of the output of the amplifier and its linearized version using the upsampled kernels $\left\{\bar{V}\left(z_{1}, \ldots, z_{p}\right)\right\}$ of the low-rate identification is depicted. The corresponding results with an additional low-rate adaptation of $\breve{V}(z)$, where the initialization is taken to be $\bar{V}(z)$, are shown in Fig. 6. The graph in Fig. 5 indicates the general result that for systems with a flat frequency response over the input signal bandwidth, such as PAs, the application of the upsampled kernels $\left\{\bar{V}\left(z_{1}, \ldots, z_{p}\right)\right\}$ already yields good linearization 


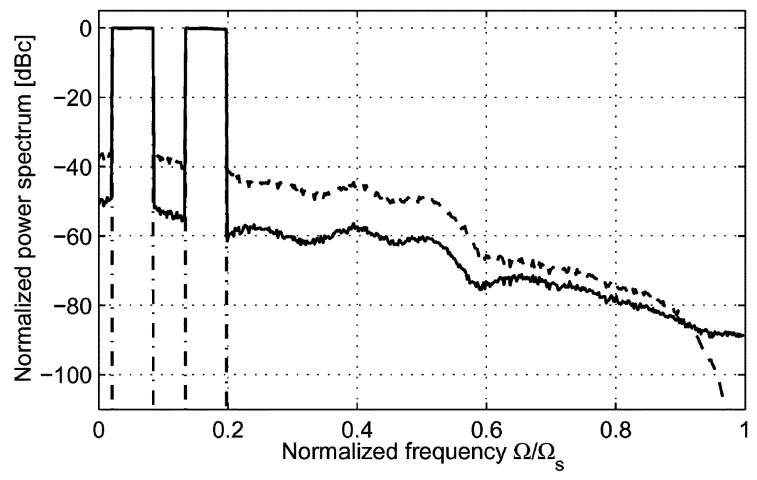

Fig. 5. Normalized power spectrum for the output of the PA V (dashed), the output of the linearization $\mathrm{V} \circ \mathrm{P}$ applying the upsampled kernels for the predistortion (solid), and the ideal linear response (dashed-dot). Results were averaged over 50 different randomly chosen DMT input signals.

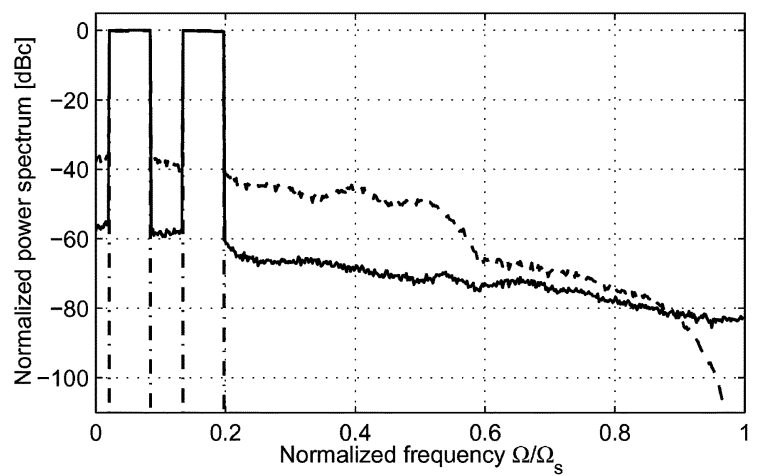

Fig. 6. Normalized power spectrum for the output of the PA $V$ (dashed), the output of the linearization $\mathrm{V}_{\circ} \mathrm{P}$ applying a low-rate adaptation for $\breve{V}(z)$ in the predistorter (solid), and the ideal linear response (dash-dotted). Results were averaged over 50 different randomly chosen DMT input signals.

performance. It is evident in Fig. 6 that a gain in linearization can be achieved by the low-rate adaptation of $\breve{V}(z)$ initialized with $\bar{V}(z)$. The underlying optimization problem for the adaptive $\breve{V}(z)$ is nonlinear because its output signal passes through the nonlinear operator $V$ to generate the low-rate error signal for the adaptation. Although good initialization is necessary for such problems in general, extensive simulation showed no critical dependence of the performance on the initialization.

\section{CONCLUSION}

Although nonlinear systems can be identified at a sampling rate that is just twice the input signal bandwidth, a sampling rate that is at least twice the output signal bandwidth is required for digital predistortion. However, we have shown that for a particular predistortion architecture and PAs with an approximately flat frequency response, it is sufficient to upsample the Volterra kernels of a low-rate identified PA and copy these high-rate kernels to the predistorter. The unwanted spectral copies of the upsampled higher order $(p \geq 2)$ kernels are masked out by the band-limited input signals. This multirate predistorter works perfectly (compared to the purely high-rate system) if the linear kernel of the PA is flat (the upsampled linear kernel is equal to the high-rate identified kernel); otherwise, we have a suboptimal solution, which in practice converges to the ideal one if we apply an additional low-rate adaptation to adjust the frequency response of the upsampled linear kernel in the predistorter over the whole frequency band.

With a typical smooth linear kernel for the PA (magnitude variation of approximately $0.5 \mathrm{~dB}$ ), we obtain an adjacent channel power suppression for the upstream signal of approximately $14 \mathrm{~dB}$ if we solely apply Volterra kernel upsampling of the low-rate identified kernels and $20 \mathrm{~dB}$ if we additionally adjust the frequency response of the upsampled linear kernel over the whole frequency band. This performance is obtained even though we only employ a low-cost low-rate ADC in the analog front end of the transmitter feedback path.

\section{ACKNOWLEDGMENT}

The authors would like to thank D. Schwingshackl, C. Vogel from Graz University of Technology, and the reviewers for their helpful comments.

\section{REFERENCES}

[1] X. Y. Gao and W. M. Snelgrove, "Adaptive linearization schemes for weakly nonlinear systems using adaptive linear and nonlinear FIR filters," in Proc. IEEE Midwest Symp. Circuits and Syst., Calgary, AB, Canada, Aug. 1990, pp. 9-12.

[2] R. Raich, H. Qian, and G. T. Zhou, "Orthogonal polynomials for power amplifier modeling and predistorter design," IEEE Trans. Veh. Technol., vol. 53, no. 5, pp. 1468-1479, Sep. 2004.

[3] J. K. Cavers, "Amplifier linearization using a digital predistorter with fast adaption and low memory requirements," IEEE Trans. Veh. Technol., vol. 37, pp. 1885-1890, Dec. 1989.

[4] V. J. Mathews and G. L. Sicuranza, Polynomial Signal Processing. New York: Wiley, 2000.

[5] L. Ding, R. Raich, and G. T. Zhou, "A Hammerstein predistortion linearization design based on the indirect learning architecture," in Proc. IEEE Int. Conf. Acoust. Speech, Signal Process., Orlando, FL, May 2002, pp. 2689-2692.

[6] W. Frank, "Sampling requirements for Volterra system identification," IEEE Signal Process. Lett., vol. 3, no. 9, pp. 266-268, Sep. 1996.

[7] P. Singerl and H. Koeppl, "A low-rate identification method for digital predistorters based on Volterra kernel interpolation," in Proc. IEEE Midwest Symp. Circuits and Syst., Cincinnati, OH, Aug. 2005, pp. $1533-1536$.

[8] R. Haber, Nonlinear System Identification-Input-Output Modeling Approach. Dordrecht: Kluwer, 1999, vol. 1.

[9] W. J. Rugh, Nonlinear System Theory. London, U.K.: Johns Hopkins Univ. Press, 1981.

[10] M. Schetzen, Volterra and Wiener Theories of Nonlinear Systems. New York: Wiley, 1980.

[11] H. Weinberger, A. Wiesbauer, M. Clara, C. Fleischhacker, T. Potscher, and B. Seger, "A 1.8 V $450 \mathrm{~mW}$ VDSL 4-band analog front end IC in $0.18 \mu \mathrm{m}$ CMOS," in Proc. IEEE Int. Solid-State Circuits Conf., San Francisco, CA, Feb. 2002, vol. 1, pp. 326-471.

[12] ETSI TM, VDSL Technical Specification-Part 1: Functional requirements European Telecommunications Standards Institute, Sophia-Antipolis, France. 